\title{
Inventory Routing Problem with Carbon Emission Consideration
}

\author{
Nur Arina Bazilah Aziz* and Choong Jing Yee \\ Department of Mathematical Sciences, Faculty of Science \\ Universiti Teknologi Malaysia, 81310 Johor Bahru, Johor, Malaysia \\ ${ }^{*}$ Corresponding author: nurarina@utm.my
}

Article history

Received: 29 December 2018

Received in revised form: 23 January 2019

Accepted: 29 January 2019

Published on line: 1 April 2019

\begin{abstract}
Inventory Routing Problem (IRP) has been continuously developed and improved due to pressure from global warming issue particularly related to greenhouse gases (GHGs) emission. The burning of fossil fuel for transportations such as cars, trucks, ships, trains, and planes primarily emits GHGs. Carbon dioxide (CO2) from burning of fossil fuel to power transportation and industrial process is the largest contributor to global GHGs emission. Therefore, the focus of this study is on solving a multi-period inventory routing problem (MIRP) involving carbon emission consideration based on carbon cap and offset policy. Hybrid genetic algorithm (HGA) based on allocation first and routing second is used to compute a solution for the MIRP in this study. The objective of this study is to solve the proposed MIRP model with HGA then validate the effectiveness of the proposed HGA on data of different sizes. Upon validation, the proposed MIRP model and HGA is applied on real-world data. The HGA is found to be able to solve small size and large size instances effectively by providing near optimal solution in relatively short CPU execution time.
\end{abstract}

Keywords Inventory routing problem; carbon emission; genetic algorithms

Mathematics Subject Classification 90B05, 90B06.

\section{Introduction}

IRP is a fundamental decision-making approach in supply chain management and has been researched and improved upon extensively, most notably since the seminar paper published by Bell et al. [1] where customers inventory level must be met under stochastics demand. In the past, classical IRP often revolves around maximizing profits and minimizing costs with some additional requirements such as travelling time or distance [2]-[3].

Over the years, IRP had been continuously developed and improved to meet various demands from current issues for instance, the global warming issue particularly related to greenhouse gases (GHGs) emission. GHGs contributes to global warming by trapping heat from leaving the atmosphere and make the planet warmer. The burning of fossil fuel for transportations such as cars, trucks, ships, trains, and planes primarily emits GHGs. Over 90 percent of the fuel used for transportation is petroleum based, which includes gasoline and diesel [4]. 


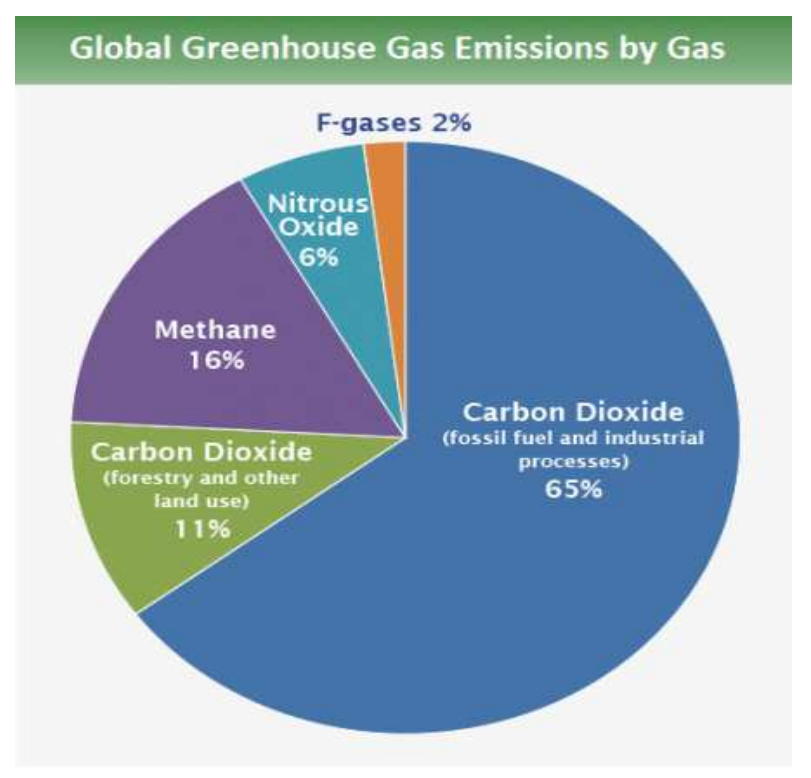

Figure 1: Global GHGs Emission [5]

Globally, the key GHGs emitted by human activities are carbon dioxide (CO2), methane (CH4), nitrous oxide (N2O) and fluorinated gases (F-gases). Figure 1 shows the contribution of each gas to the global GHGs emission. It is obvious that CO2 from fossil fuel and industrial process is the largest contributor to global GHGs emission at a total of 65 percent. Therefore, it is crucial to tackle on reducing the amount of CO2 gases in the atmosphere to prevent global warming from getting worse.

\section{U.S. Carbon Dioxide Emissions, By Source}

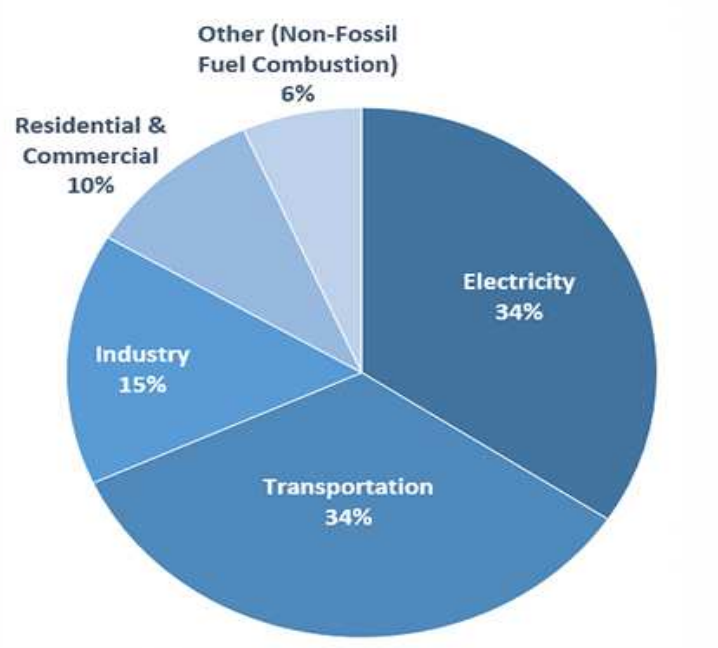

Figure 2: US CO2 Emission by Source [6]

Other than that, from Figure 2, in United States (US), transportation and electricity are the largest share of $\mathrm{CO} 2$ at 34 percent. Which means that, focusing on reducing CO2 emission 
in the transportation sector might result in great benefits to the environment. The issue of reducing $\mathrm{CO} 2$ emission in transportation sector gave motivation to researchers to study on the management of transportation in supply chain activities which leads to the improvement on the classical IRP model by considering carbon emission in the model.

The focus of this study is to solve a multi-period inventory routing problem (MIRP) involving carbon emission consideration based on carbon cap and offset policy. HGA based on allocation first and routing second is used to compute a solution for the MIRP in this study. The HGA is a combined algorithm of GA and double sweep algorithm (DSW) with the former is used for allocation decisions, while the latter is used for routing decisions. The supply chain involved in this study is an inbound product collection network with one depot, a set of geographically dispersed suppliers and one assembly plant with deterministic and time-varying demands. The transportation cost, fuel consumption cost and inventory holding cost are fixed. Fuel consumption is used to generate the value of carbon emissions. The expected solution will display the best supplier allocation choice and product collection route which results in minimal system's total cost.

\section{Inventory Routing Problem with Carbon Emission Consideration}

One of the first study on IRP involving carbon emission consideration was done by Sourirajan et al. [7]. They presented the IBM Carbon Analyzer Tool, a software solution that models and quantifies carbon emissions and explores ways to reduce carbon emissions associated with the support logistics for an assembly manufacturing operation through advanced analytics. The tool has four analytical modules. One of the module related to IRP was the scenario analysis module which explores various consolidation policies to minimize transportation, inventory, and carbon costs, subject to inventory availability requirements.

However, study on sustainable IRP had been scarce until Mirzapour et al. [8] addressed a multi-product multi-period Inventory Routing Problem where multiple capacitated vehicles distribute products from multiple suppliers to a single plant to meet deterministic and time varying demand of each product over a finite planning horizon. GHG emission level was also incorporated into the model and the proposed model is a mixed-integer linear program and solved by CPLEX. The GHG emission level was measured by travel distance. Sazvar et al. [9] developed a stochastic mathematical model and proposed a new replenishment policy in a centralized supply chain for perishable products with uncertain demand. Several transportation vehicles producing various GHG levels are considered. The best transportation vehicles and inventory policy are determined by finding a balance between supply chain cost and environmental criteria.

\section{The Mathematical Formulation}

\subsection{Assumptions}

This study focuses on the inbound product collection system consisting of an assembly plant, a depot and $N$ suppliers. At the beginning of the product collection process, vehicles depart the depot and collect products from the suppliers then deliver these products to the assembly 
plant. By the end of the product collection process, vehicles must return to depot after delivering all the products collected from suppliers to the assembly plant to complete the delivery. Assumptions made in the proposed mathematical model are as follows:

- Each supplier provides one type of product. In the first period, all the products are ready for collection.

- The demand at the assembly plant is deterministic but varies in each period.

- Stockouts at the assembly plant is not permitted.

- Inventory holding costs are only considered in assembly plan and varies according to products. The initial inventory level of products is zero.

- Vehicles are homogeneous and capacitated. The number of vehicles is unlimited.

- Split pick up is not allowed.

- The carbon emission regulations are imposed on each period during the time horizon.

\subsection{Mathematical Model}

The proposed mathematical model uses the following notations:

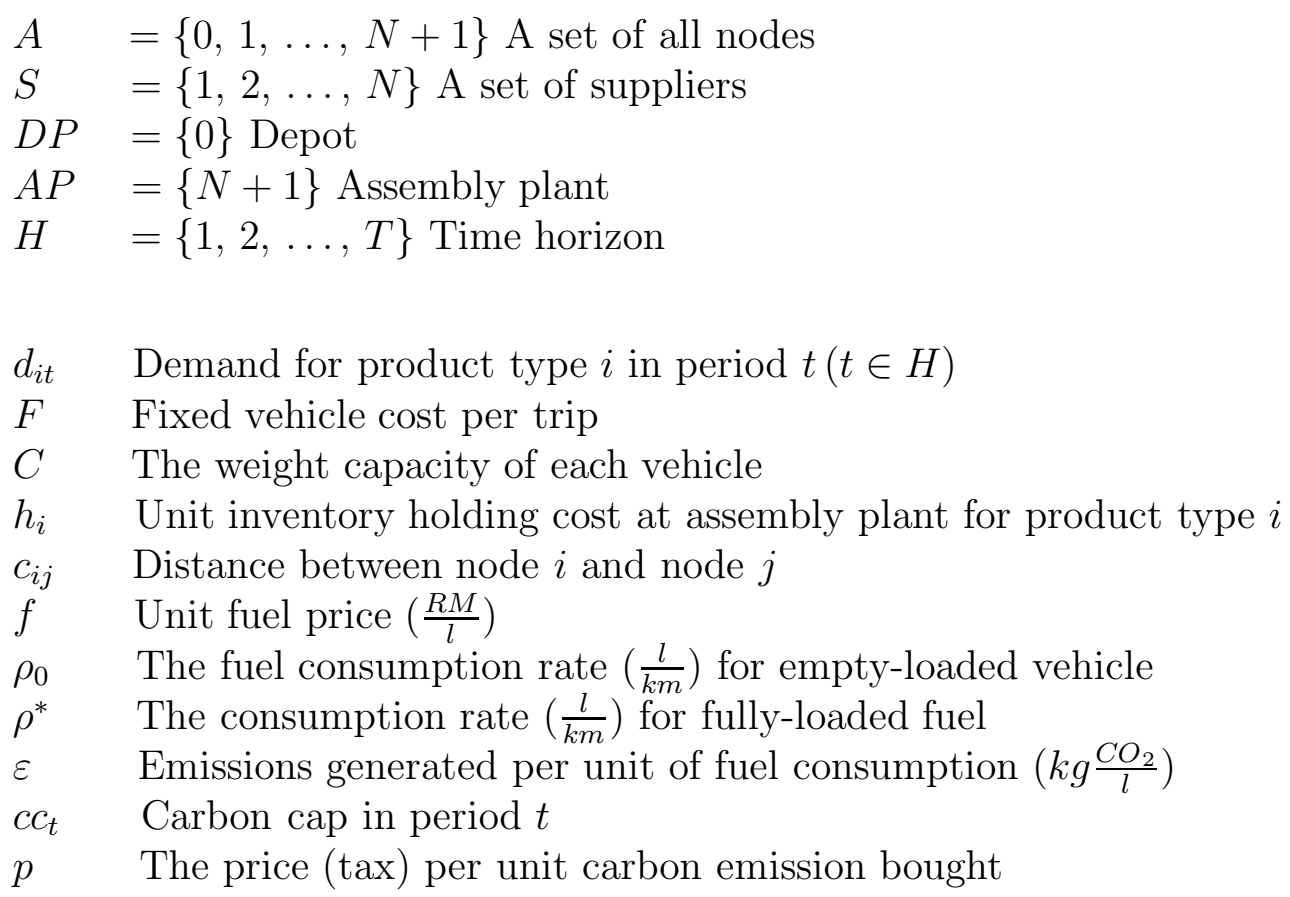

\section{Decision variables}

$x_{i j t} \quad x_{i j t}= \begin{cases}1, & \text { if } \operatorname{arc}(i, j) \text { is traversed by a vehicle in period } t . \\ 0, & \text { otherwise. }\end{cases}$

$\omega_{i j t} \quad$ The total product weight carried by a vehicle through arc $(i, j)$ in period $t$

$q_{i t} \quad$ The product quantity picked up at supplier $i$ in period $t$ 
$I_{i t} \quad$ The inventory level of product $i$ at the assembly plant at the end of period $t$

$f c_{i j t} \quad$ Fuel consumption from node $i$ to node $j$ in period $t$

$e_{t}^{+} \quad$ Amount of carbon emission credits purchased in period $t$

The MIRP model proposed in this study was introduced by Cheng et al. [10]. The supply chain network is imposed with a carbon cap in each period under a carbon cap and offset policy. This policy places a cap on the amount of carbon emission permitted for each period and allows the purchase of extra carbon emission credits in the carbon offset market if the carbon emission of an organization exceeds the carbon cap imposed. Carbon emission depends on the fuel consumption according to Zhang et al. [11] and Cachon [12], therefore the carbon emission is measure based on the fuel consumption. The computation of fuel consumption is discussed in the following subtopic followed by the formulation of MIRP model.

$$
\begin{aligned}
\operatorname{Min} Z= & \sum_{i \in S} \sum_{t \in H} h_{i} I_{i t}+\sum_{t \in H} \sum_{j \in S} F x_{0 j t} \\
& +f\left(\sum_{t \in H} \sum_{i \in S \cup D P} \sum_{j \in S \cup A P}\left(\rho_{0} c_{i j} x_{i j t}+\beta c_{i j} w_{i j t}\right)+\sum_{t \in H} \sum_{j \in S} \rho_{0} c_{N+1,0} x_{0 j t}\right)+\sum_{t \in H} p e_{t}^{+}
\end{aligned}
$$

The objective function aims to minimize the total cost of the system which include the inventory holding costs, fixed transportation costs, fuel consumption costs and the cost of purchasing the extra emission credits. From objective function above, $\sum_{i \in S} \sum_{t \in H} h_{i} I_{i t}$ is the inventory holding cost, $\sum_{t \in H} \sum_{j \in S} F x_{0 j t}$ is the fixed transportation cost, $f\left(\sum_{t \in H} \sum_{i \in S \cup D P} \sum_{j \in S \cup A P}\left(\rho_{0} c_{i j} x_{i j t}+\beta c_{i j} w_{i j t}\right)+\right.$ $\left.\sum_{t \in H} \sum_{j \in S} \rho_{0} c_{N+1,0} x_{0 j t}\right)$ is the fuel consumption cost and $\sum_{t \in H} p e_{t}^{+}$is the carbon emission cost. The constraints involved in this problem are as below:

subject to

$$
\begin{array}{ll}
I_{i t}=I_{i(t-1)}+q_{i t}-d_{i t} & \forall i \in S, \forall t \in H \\
\sum_{j \in S \cup D P} \omega_{j i t}+q_{i t}=\sum_{j \in S \cup A P} \omega_{i j t} & \forall i \in S, i \neq j, \forall t \in H \\
\sum_{j \in S \cup D P} x_{j i t}=\sum_{j \in S \cup A P} x_{i j t} & \forall i \in S, \forall t \in H \\
\sum_{j \in S \cup D P} x_{i j t} \leq 1 & \forall i \in S, \forall t \in H \\
\sum_{i \in S} x_{0 i t}=\sum_{i \in S} x_{i(N+1) t} & \forall t \in H
\end{array}
$$

Constraint (1) is the inventory balance equation for each product at the assembly plant. Constraint (2) is the product flow balance equation, which guarantees the flow balance at each 


$$
\begin{array}{ll}
\varepsilon\left(\sum_{i \in S \cup D P} \sum_{j \in S \cup A P}\left(\rho_{0} c_{i j} x_{i j}+\beta c_{i j} w_{i j t}\right)+\sum_{j \in S} \rho_{0} c_{N+1,0} x_{0 j t}\right) \leq c c_{t}+e_{t}^{+} \quad \forall t \in H \\
\omega_{i j t} \leq C x_{i j t} & \forall i \in S, \forall j \in S \cup A P, \forall t \in H \\
x_{0(N+1) t}=0 & \forall t \in H \\
x_{i 0 t}=0 & \forall i \in S, \forall t \in H \\
x_{(N+1) j t}=0 & \forall i \in S, \forall t \in H \\
x_{i i t}=0 & \forall i \in A, \forall t \in H \\
\omega_{0 i t}=0 & \forall i \in S, \forall t \in H \\
I_{i 0}=0 & \forall i \in S \\
w_{i j t} \geq 0 & \forall i, j \in A, \forall t \in H \\
w_{i j t} \geq C x_{i j t}+\omega_{i j t}-C & \forall i, j \in A, \forall t \in H \\
w_{i j t} \leq \omega_{i j t} & \forall i, j \in A, \forall t \in H \\
w_{i j t} \leq C x_{i j t} & \forall i, j \in A, \forall t \in H \\
x_{i j t}=\{0,1\} & \forall i, j \in A, \forall t \in H \\
q_{i t}, I_{i t}, \omega_{i j t}, e_{t}^{+} \geq 0 & \forall i, j \in A, \forall t \in H
\end{array}
$$

supplier and eliminates all subtours. Constraint (3) guarantees that the number of vehicles leaving a supplier is equal to the number of vehicles arriving at the supplier. Constraint (4) ensures that split pick up is not allowed. Constraint (5) ensures that the number of vehicles leaving a depot is equals to the number of vehicles arriving at the assembly plant. Constraint (6) ensures the total emission in each period does not exceed the sum of the cap and the purchased extra emission credits. Constraint (7) is to make sure the vehicle capacity is not violated. Constraint (8) to constraint (9) are the impossible arc. Constraint (10) guarantees the weight of vehicle from depot to suppliers is zero. Constraint (11) is to ensure the initial inventory level of all products at the assembly plant is zero. Constraint (12) to constraint (13) are obtained after the original mathematical model is linearized. Constraint (14) to constraint (19) are the types of variables in this model.

\section{Hybrid Genetic Algorithms}

Solving the MIRP for large data size is computationally expensive. A metaheuristic algorithm such as HGA is necessary to find a satisfactory solution for MIRP which is a large and complex NP-hard problem in a reasonable amount of time. The proposed HGA will decide whether a vehicle will collect products from a supplier in each period. As for the best product collection route a modified sweep algorithm introduced by Lee et al. [13] also known as the DSW is used for decisions in clustering and routing. The operators used for genetic algorithm are the 
crossover operator and mutation operator. The proposed HGA uses the following notations:

$K \quad$ Population size

$G^{\prime} \quad$ The number of generations

$P_{c} \quad$ Crossover rate

$P_{m} \quad$ Mutation rate

$l \quad$ Chromosome

Genetic algorithm comprises of a set of population which is made up of $K$ chromosomes, each carries a solution to the problem. The chromosome representation used in this study is a binary matrix of with $N \times T$ genes [14]. $N$ represents the number of suppliers while $T$ is the number of periods. Each gene indicates whether a supplier will be visited in each period. The genes in a chromosome is represented as below:

$$
C h_{i t}^{l}= \begin{cases}1, & \text { supplier } i \text { is visited in period } t \text { for chromosome } l \\ 0, & \text { otherwise. }\end{cases}
$$

The binary chromosome representation matrix is used to determine the quantity of product which will be collected from a supplier in a period. For instance, if a supplier is visited on a period $t$ and will be visited again on period $t^{\prime}\left(t^{\prime}>t\right)$, the quantity of products collected on period $t$ is the sum of demands from period $t$ to period $t^{\prime}-1$. Table 1 shows an example for the binary matrix representation and the corresponding product collection matrix with $5 \times 5$ genes. For instance, supplier 2 will be visited in period $1\left(C h_{21}^{l}=1\right)$ and will only be visited again in period $3\left(C h_{23}^{l}=1\right)$. Therefore, the products collected from supplier 2 in period 1 is the sum of demand from period 1 to period $2(4+2=6)$ while the products collected from supplier 2 in period 3 is the sum of demand from period 3 to period $5(1+1+1=3)$.

Table 1: Example of Binary Matrix Representation and Product Collection Matrix.

\begin{tabular}{|l|c|ccccc|}
\cline { 2 - 7 } \multicolumn{1}{c|}{} & \multirow{2}{*}{ Supprier } & \multicolumn{5}{c|}{ Period } \\
\cline { 2 - 7 } & & $\mathbf{1}$ & $\mathbf{2}$ & $\mathbf{3}$ & $\mathbf{4}$ & $\mathbf{5}$ \\
\hline Chromosome Representation & $\mathbf{1}$ & $\mathbf{1}$ & $\mathbf{0}$ & $\mathbf{1}$ & $\mathbf{1}$ & $\mathbf{1}$ \\
& 2 & 1 & 0 & 1 & 0 & 0 \\
& $\mathbf{3}$ & $\mathbf{1}$ & $\mathbf{1}$ & $\mathbf{0}$ & $\mathbf{1}$ & $\mathbf{1}$ \\
& $\mathbf{4}$ & $\mathbf{1}$ & $\mathbf{0}$ & $\mathbf{1}$ & $\mathbf{0}$ & $\mathbf{0}$ \\
& $\mathbf{5}$ & $\mathbf{1}$ & $\mathbf{0}$ & $\mathbf{0}$ & $\mathbf{1}$ & $\mathbf{1}$ \\
\hline Demand Matrix & $\mathbf{1}$ & $\mathbf{5}$ & $\mathbf{1}$ & $\mathbf{4}$ & $\mathbf{3}$ & $\mathbf{4}$ \\
& 2 & 4 & 2 & 1 & 1 & 1 \\
& $\mathbf{3}$ & $\mathbf{1}$ & $\mathbf{5}$ & $\mathbf{5}$ & $\mathbf{5}$ & $\mathbf{5}$ \\
& $\mathbf{4}$ & $\mathbf{2}$ & $\mathbf{2}$ & $\mathbf{5}$ & $\mathbf{3}$ & $\mathbf{2}$ \\
& $\mathbf{5}$ & $\mathbf{2}$ & $\mathbf{3}$ & $\mathbf{2}$ & $\mathbf{5}$ & $\mathbf{3}$ \\
\hline Product Collection Matrix & $\mathbf{1}$ & $\mathbf{6}$ & $\mathbf{0}$ & $\mathbf{4}$ & $\mathbf{3}$ & $\mathbf{4}$ \\
& 2 & 6 & 0 & 3 & 0 & 0 \\
& $\mathbf{3}$ & $\mathbf{1}$ & $\mathbf{1 0}$ & $\mathbf{0}$ & $\mathbf{5}$ & $\mathbf{5}$ \\
& $\mathbf{4}$ & $\mathbf{4}$ & $\mathbf{0}$ & $\mathbf{1 0}$ & $\mathbf{0}$ & $\mathbf{0}$ \\
& $\mathbf{5}$ & $\mathbf{7}$ & $\mathbf{0}$ & $\mathbf{0}$ & $\mathbf{5}$ & $\mathbf{3}$ \\
\hline
\end{tabular}

In each generation, chromosomes will undergo fitness test to select which chromosomes to undergo genetic evolution through the crossover and mutation process. After going through 
crossover and mutation, new chromosomes will replace the old ones to create a new set of population for the next generation. The chromosomes will evolve until the $G^{\prime}$ number of generations is reached and the best chromosome among the population in the last generation is the near optimal solution for the problem. The steps for HGA algorithm are as follows:

Step 1: Randomly generate initial population.

At the beginning of the stage, a set of initial population in binary matrix is generated randomly. If the demand for product $i$ in period $t=1$ is nonzero, set $C h_{i 1}^{l}=1$; otherwise set $C h_{i 1}^{l}=0$, for stockout prevention. If supplier $i$ has been visited in period $t$, randomly generate $C h_{i t^{\prime}}^{l}=0$ or $C h_{i t^{\prime}}^{l}=1$ for $t^{\prime}\left(t^{\prime}>t\right)$; otherwise set $C h_{i t^{\prime}}^{l}=1$.

Step 2: Compute the product collection matrix.

After generating the initial population, the corresponding product collection matrix can be computed. If supplier $i$ is visited in period $t(t \neq T)$ and will be visited again in later period $t^{\prime}\left(t^{\prime}>t\right)$, then the products to be collected in period $t$ is the sum of demand from period $t$ to period $t^{\prime}-1$. Meanwhile, if supplier $i$ is visited in period $t(t \neq T)$ and will not be visited again in later period, then the products to be collected in period $t$ is the sum of demand from period $t$ to period T. If supplier $i$ is visited in period $T$, then the products to be collected in period $T$ is the demand for product type $i$ in period $T$. Please refer to Table 1 on the previous page for the example on how product collection matrix is computed.

Step 3: Perform DSW to compute the routing for product collection.

The product collection matrix from Step 2 does not show how suppliers will be visited in each period. To minimize the travelling distance which will affect the fuel consumption and carbon emission, DSW is applied to the product collection matrix.

(a) For each period $t=1,2, \ldots, T$, arrange the suppliers $S=\{1,2, \ldots, N\}$ and the assembly plant, $A P$ around the depot, $D P$ such that the $y$-coordinate of the $A P$ is the same as the $y$-coordinate of the $D P$. This can be done by rotating the nodes on a cartesian plane while retaining the distances between each other. The rotation is done by converting the coordinates $(x, y)$ of the nodes to polar coordinates $(r, \theta)$. Then rotate all the nodes except $D P$ in an angle so that $A P$ will have the same $\theta$ as $D P$.

(b) Sort the suppliers in ascending order according to their new $y$-coordinate values. Let $S_{i}$ be the $i^{\text {th }}$ supplier after the sort.

(c) Set $i=1$ and $k=1$, where $k$ is cluster. Open a route $R_{k}=\{\}$ and set $q_{k}=0$, where $q_{k}$ is the total pick-up quantity assigned to cluster $k$. If $q_{k}+d_{i t} \leq C$, assign $S_{i}$ to route $R_{k}$, set $q_{k}=q_{k}+d_{i t}$. Proceed with $i=2,3, \ldots, N$, If $q_{k}+d_{i t}>C$, set $k=k+1$ to open a new route $R_{k}=\{\}$, assign $S_{i}$ to $R_{k}$. Reset $k=1$ and repeat the clustering process until all the suppliers are assigned to a route.

(d) Sort the suppliers within route $R_{k}$ according to their $x$-coordinate values in ascending order. Let $S_{i}^{k}$ be the $i^{\text {th }}$ supplier in route $R_{k}$ after the sort. Form a route that starts at the $D P$, visit all the suppliers in $R_{k}$, deliver the products to $A P$ and returns to $D P$. Then go to Step 4. 
Step 4: Compute total costs and fitness value for chromosome selection.

The numbers obtained from Step 1 to Step 3 are plucked into equation (3) to compute the total cost of the system. The fitness value is based on the total costs of each chromosome in a population. A roulette wheel selection method is used for the chromosome selection.

Step 5: Perform crossover and mutation operator.

The crossover operator is used to mate one pair of chromosomes to produce two new offspring. The two selected chromosomes in Step 4 are crossed with a bitwise AND to obtain Child 1 and a bitwise OR to obtain Child 2.

Mutation operator is performed after crossover to increase the diversity of the population. Firstly, a chromosome is randomly selected from current population. Secondly, a of matrix with same size consisting of random numbers is generated. These numbers are then compared to the mutation rate, $P_{m}$. If the number is lesser than $P_{m}$, the corresponding gene will be mutated. If the gene is " 1 ", then transform it to 0 ; otherwise, if it is " 0 ", then transform it to " 1 ".

Step 6: Repeat Step 2 to Step 5 until the maximum number of generations is attained.

The chromosome which give the best result in the last generation is the solution of the problem.

\section{$5 \quad$ Results and Discussion}

Three groups of data sets are applied to the proposed MIRP model and solved using HGA. The solutions from these data are compared to existing exact solution to validate the effectiveness of the proposed model and algorithm. Data sets used in this study are based on [13]. The smallest group of data consists of $S 3 H 3, S 3 H 14, S 5 H 5$, and $S 5 H 14$, the medium group of data group consists of $S 9 H 5, S 9 H 14, S 12 H 5, S 12 H 14$, while the largest group of data consists of $S 20 H 15, S 2 H 21, S 50 H 15, S 50 H 21$. Each instance is run 10 times, the best solution and average CPU execution time is recorded.

Table 2 shows the comparison between the solutions obtained from the proposed algorithm and exact solutions. The last column in Table 2 shows the solution gap percentage between CPLEX's best integer and HGA. Observe that the CPU execution time took by HGA to find a solution increases slightly as the size of data increases while CPLEX is able to find optimal solution in a short amount time for smaller data sizes but unable to do so as the size of the data increases starting from the $S 9 H 14$ data set. This shows that exact method is capable in solving smaller set of data, but it may not be suitable for bigger data which makes it incapable in solving real world problem with big data set. On the other hand, the proposed HGA might not be as efficient as CPLEX in solving smaller size data as the CPU execution time are slightly longer than CPLEX for $S 3 H 3, S 3 H 14, S 5 H 5$ and $S 9 H 5$ data set. However, HGA still managed to produce a solution in less than 1 minute (60 s). Hence, to solve a problem bigger than $S 9 H 14$ where the CPLEX will be too time consuming, HGA is a more suitable choice as the CPU execution time is significantly faster than CPLEX. 
Table 2: Comparison of Results between HGA and CPLEX

\begin{tabular}{|c|c|c|c|c|c|}
\hline & \multicolumn{2}{|c|}{ CPLEX (Exact Method) } & \multicolumn{2}{|c|}{ HGA (Heuristic Method) } & \multirow[b]{2}{*}{ Gap (\%) } \\
\hline Data Groups & Best Integer & $\mathrm{CPU}$ (s) & Solution & $\mathrm{CPU}$ (s) & \\
\hline S3H3 & 194.13 & 0.40 & 196.59 & 16.65 & 1.27 \\
\hline S3H14 & 955.03 & 1.20 & 970.24 & 17.24 & 1.59 \\
\hline S5H5 & 402.36 & 1.43 & 403.99 & 16.51 & 0.40 \\
\hline S5H14 & $1,234.37$ & 773.49 & $1,286.33$ & 18.41 & 4.21 \\
\hline S9H5 & $1,175.70$ & 4.18 & $1,312.91$ & 18.70 & 11.67 \\
\hline S9H14 & $3,682.88$ & $3,600.00$ & $4,200.79$ & 22.37 & 14.06 \\
\hline S12H5 & $1,733.64$ & $3,600.00$ & $1,982.96$ & 32.30 & 14.38 \\
\hline S12H14 & $5,212.27$ & $3,600.00$ & $6,160.91$ & 42.38 & 18.20 \\
\hline S2OH15 & $5,121.19$ & $3,600.00$ & $6,296.93$ & 63.35 & 22.96 \\
\hline S2OH21 & $7,280.94$ & $3,600.00$ & 9,158.35 & 79.56 & 25.79 \\
\hline S5OH15 & $15,042.42$ & $3,600.00$ & $15,532.40$ & 183.85 & 3.26 \\
\hline S50H21 & 22,071.99 & $3,600.00$ & $22,757.80$ & 253.92 & 3.11 \\
\hline
\end{tabular}

\section{Acknowledgement}

The author would like to acknowledge the Ministry of Education Malaysia and Research Management Centre-UTM, Universiti Teknologi Malaysia (UTM) for the financial support through High Impact Research grant with vote number 04G43 for this research.

\section{References}

[1] Bell, W. J., Dalberto, L. M., Fisher, M. L., Greenfield, A. J., Jaikumar, R., Kedia, P., Mack, R. G. and Prutzman, P. J. Improving the distribution of industrial gases with an on-line computerized routing and scheduling optimizer. Interfaces. 1983. 13: 4-23.

[2] Li, K. P., Chen, B., Sivakumar, A. I. and Wu, Y. An inventory-routing problem with the objective of travel time minimization. European Journal of Operational Research. 2014. 236: 936-945.

[3] Madadi, A., Kurz, M. E. and Ashayeri, J. Multi-level inventory management decisions with transportation cost consideration. Transportation Research Part E-Logistics and Transportation Review. 2010. 46: 719-734.

[4] Intergovernmental Panel on Climate Change (IPCC). Chapter 5: Transport and its infrastructure - AR4 WGIII. 2007. [online] Available at: http://www.ipcc.ch/publications_and_data/ar4/wg3/en/ch5.html [Accessed 21 May 2018].

[5] Intergovernmental Panel on Climate Change (IPCC). Fifth Assessment Report - Mitigation of Climate Change. 2014.[online] Available at: https://www.ipcc.ch/report/ar5/wg3/ [Accessed 21 May 2018]. 
[6] U.S. Environmental Protection Act (EPA). Inventory of U.S. Greenhouse Gas Emissions an Sinks. 2018. [online] Available at:

https://www.epa.gov/sites/production/files/2018-01/documents/2018_complete_report.pdf [Accessed 21 May 2018].

[7] Sourirajan, K., Centonze, P., Helander, M. E., Katircioglu, K., Ben-Hamida, M. and Boucher, C. Carbon management in assembly manufacturing logistics. Ibm Journal of Research and Development. 2009. 53(3): 8-1.

[8] Mirzapour Al-E-Hashem, S. M. J. and Rekik, Y. Multi-product multi-period inventory routing problem with a transshipment option: A green approach. International Journal of Production Economics. 2014. 157: 80-88.

[9] Sazvar, Z., Mirzapour AL-E-Hashem, S. M. J., Baboli, A. and Akbari Jokar, M. R. A bi-objective stochastic programming model for a centralized green supply chain with deteriorating products. International Journal of Production Economics.2014. 150: 140-154.

[10] Cheng, C., Qi, M. Y., Wang, X. Y. and Zhang, Y. Multi-period inventory routing problem under carbon emission regulations. International Journal of Production Economics. 2016. 182: $263-275$.

[11] Zhang, S. Z., Lee, C. K. M., Choy, K. L., Ho, W. and Ip, W. H. Design and development of a hybrid artificial bee colony algorithm for the environmental vehicle routing problem. Transportation Research Part D-Transport and Environment. 2014. 31: 85-99.

[12] Cachon, G. P. Retail store density and the cost of greenhouse gas emissions. Management Science. 2014. 60: 1907-1925.

[13] Lee, C. G., Bozer, Y. A. and White III, C. A heuristic approach and properties of optimal solutions to the dynamic inventory routing problem. Working Paper. Toronto, Canada: University of Toronto. 2003.

[14] Moin, N. H., Salhi, S. and Aziz, N. A. B. An efficient hybrid genetic algorithm for the multi-product multi-period inventory routing problem. International Journal of Production Economics. 2011. 133: 334-343. 